\title{
Novel targeted nuclear imaging agent for gastric cancer diagnosis: glucose-regulated protein 78 binding peptide-guided "'In-labeled polymeric micelles
}

\author{
This article was published in the following Dove Press journal: \\ International Journal of Nanomedicine \\ 9 April 2013 \\ Number of times this article has been viewed
}

\author{
Chun-Chia Cheng ${ }^{1,2, *}$ \\ Chiung-Fang Huang ${ }^{3,4, *}$ \\ Ai-Sheng $\mathrm{Ho}^{5}$ \\ Cheng-Liang Peng ${ }^{6}$ \\ Chun-Chao Chang 7,8 \\ Fu-Der Mai ${ }^{1,9}$ \\ Ling-Yun Chen ${ }^{10}$ \\ Tsai-Yueh Luo ${ }^{2}$ \\ Jungshan Chang 1,11,12
}

'Graduate Institute of Medical Sciences,

Taipei Medical University, Taipei, ${ }^{2}$ Institute of Nuclear Energy Research, Atomic

Energy Council, Taoyuan, ${ }^{3}$ School of Dental

Technology, Taipei Medical University, Taipei,

${ }^{4}$ Division of Family and Operative Dentistry,

Department of Dentistry, Taipei Medical

University Hospital, Taipei, ${ }^{5}$ Division of

Gastroenterology, Cheng Hsin General

Hospital, Taipei, 'Institute of Biomedical

Engineering, National Taiwan University,

Taipei, ${ }^{7}$ Division of Gastroenterology

and Hepatology, Department of Internal

Medicine, Taipei Medical University

Hospital, Taipei, ${ }^{8}$ Department of Internal

Medicine, Taipei Medical University, Taipei,

${ }^{9}$ Department of Biochemistry, Taipei

Medical University, Taipei, ${ }^{10}$ Institute

of Biochemistry and Biotechnology,

Chung Shan Medical University, Taichung,

"Neuroscience Research Center, Taipei

Medical University Hospital, Taipei,

${ }^{12}$ Research Center for Biomedical Implants

and Microsurgery Devices, Taipei Medical

University, Taipei, Taiwan

*These authors contributed equally to this work

Correspondence: Jungshan Chang No 250 Wusing Street, Sinyi District, Taipei II03I, Taiwan

Tel +88622736 I66I ext 3423

Fax +886 223778620

Email js.chang@tmu.edu.tw
Abstract: Increased expression of cellular membrane bound glucose-regulated protein 78 (GRP78) is considered to be one of the biomarkers for gastric cancers. Therefore, peptides or molecules with specific recognition to GRP78 can act as a guiding probe to direct conjugated imaging agents to localized cancers. Based on this rationale, GRP78-guided polymeric micelles were designed and manufactured for nuclear imaging detection of tumors. Thiolated GRP78 binding peptide (GRP78BP) was first labeled with maleimide-terminated poly(ethylene glycol)$\operatorname{poly}(\varepsilon$-caprolactone) and then mixed with diethylenetriaminepentaacetic acid (DTPA)-linked poly(ethylene glycol)-poly( $\varepsilon$-caprolactone) to form DTPA/GRP78BP-conjugated micelles. The coupling efficiency of micelles with radioisotope indium-111 ( $\left.{ }^{111} \mathrm{In}\right)$ was measured and analyzed by instant thin layer chromatography. The coupling efficiency of DTPA-conjugated micelles and DTPA/GRP78BP-conjugated micelles with ${ }^{111}$ In was $85 \%$ and $93 \%$, respectively. For characterization and trace imaging, the radioisotope ${ }^{111}$ In-targeting tumors were detected and imaged in a xenograft murine model using nano single photon emission computed tomography/ computed tomography. The results revealed that the radioactive intensity measured in the animals administered with GRP78BP-guided ${ }^{111}$ In-labeled micelles was statistically higher than that in animals administered with ${ }^{111}$ In-labeled micelles, demonstrating that GRP78BP more than doubled the accumulation of micelles to the tumor tissue $(P<0.05)$. The results indicate that the gastric cancer biomarker GRP78 is a probing target in the application of nuclear imaging for tumor diagnosis. This novel GRP78BP-guided micelle agent may be applied in clinical practice to complement the histological diagnosis.

Keywords: biomarker, glucose-regulated protein 78, nuclear imaging, gastric cancer, micelles

\section{Introduction}

Pathological examination based on gastroscopy is the gold standard for gastric cancer (GC) diagnosis in clinical practice. However, there remain some limitations in making an accurate diagnosis due to, for example, sampling errors and the diagnostic variations derived from personal skill. ${ }^{1}$ In addition to invasive tissue biopsy, noninvasive functional imaging tools such as magnetic resonance imaging, single photon emission computed tomography (SPECT), positron emission tomography, and optical fluorescence imaging are used to detect molecular abnormalities of tumors in clinical practice. ${ }^{2-6}$ These realtime imaging tools allow physicians to study and determine the tumorigenic progression of patients with rapid and more precise diagnosis, which can reduce the diagnostic inaccuracy derived from the inconvenient histological examinations. 
Among these imaging technologies and tools, nuclear imaging provides the strongest sensitivity in signal capturing because it has a radiation ray with the highest penetration rate to biological tissues. Moreover, many studies suggest that a combined tool consisting of the nuclear imaging from radioisotopes and anatomical images from computed tomography (CT) in a single imaging device (SPECT/CT and positron emission tomography/CT) is useful in diagnosis because of dual validation and minimized false signals. ${ }^{6-8}$ In general, it is complementary rather than competition between different imaging acquiring techniques. Therefore, developing an imaging reagent that can be detected and reported simultaneously by positron emission tomography and CT for tumor imaging is certainly useful to validate the accuracy in detection. Therefore, the development of multifunctional imaging agents that can provide more detailed evidence to improve diagnostic accuracy for clinical physician-related professionals is encouraged.

Recently, polymeric nanoparticles (NPs) such as liposomes, nanogold particles, dendrimers, and micelles have been used as drug carriers in cancer diagnostics and therapeutics due to their high enhanced permeability and retention effect, which leads to increased drug accumulation and spread into the tumor parenchyma., ${ }^{3,-13}$ It has been reported that synthetic biocompatible polymer NPs can also prolong the half-life of drugs in circulation coupled with a reduction in the rapid renal clearance of soluble proteins by the reticuloendothelial system. ${ }^{2,14}$ Therefore, imaging reagents or anticancer drugs equipped with NP carriers can gain more accessibility into tumors. Polymeric micelles composed of amphiphilic copolymers can encapsulate various insoluble molecules (eg, hydrophobized doxorubicin) and can be labeled with specific tumor binding probes (eg, a tumor binding peptide). ${ }^{14-17}$ Moreover, phospholipid micelles with unique properties, including good biocompatibility and high stability both in vitro and in vivo, suggest they are good candidates for a drug carrier shuttle in clinical practice. ${ }^{18,19}$

Previously, it was found that glucose-regulated protein 78 (GRP78) is a reliable GC biomarker with increased expression level on the GC cell surface, and the specific GRP78 binding peptide (GRP78BP), WIFPWIQL, can enhance the accumulated efficacy of polymeric micelles within cancer cells in vitro and in vivo. ${ }^{14,20}$ To the authors' knowledge, GRP78 is an endoplasmic reticulum protein and functions as a refolding protein, which would translocate to the plasma membrane in tumor cells as a tumor target. ${ }^{21}$ Therefore, enhanced drugs targeting to GRP78 of tumor cells may be useful in nuclear imaging during diagnosis, leading to improved diagnostic accuracy.
In a previous study, rhenium-188 (half-life of 16.9 hours) an isotope for both therapeutic and diagnostic purposes - was conjugated with micelles for tumor detection. ${ }^{15}$ However, rhenium-188 emits a $\beta$-ray that would damage the normal organs such as the liver and spleen, which accumulate a large amount of micelles. To lessen the tissue damage, indium-111 ( $\left.{ }^{111} \mathrm{In}\right)$ was chosen for the current study (only emits $\gamma$-ray with a halflife of 2.83 days) as a diagnostic radiotracer to conjugate with polymeric micelles. Moreover, the hydrophilic radiotracer conjugated with micelles can reduce the blood clearance rate through the renal clearance route. With the assistance of a tumor-specific binding probe, it enhanced micelles to target tumors for intracellular delivery of the imaging agent, ${ }^{6,22,23}$ improving the diagnostic efficacy of imaging agents.

The aim of this study was to rationally design and construct polymeric micelles carrying a tumor-directing peptide (GRP78BP) and the isotope chelator diethylenetriaminepentaacetic acid (DTPA) to generate a novel nuclear imaging agent during tumor diagnosis.

\section{Material and methods}

\section{Tumor xenograft mice}

Animal experimentation was performed according to the approved procedures of the Institute of Nuclear Energy Research, Atomic Energy Council (Taoyuan, Taiwan). Tumor xenografts were established by subcutaneously injecting $2 \times 10^{6}$ human GC cells (MKN45) into the legs of nude 6-8-week-old mice (BioLASCO Taiwan Co, Ltd, Taiwan). The tumor imaging experiment was performed 2 weeks after the injection of tumor cells.

\section{Synthesis of methoxy poly(ethylene glycol)-poly( $\varepsilon$-caprolactone) (MPEG-PCL) and maleimide-terminated PEG-PCL (Mal-PEG-PCL)}

The synthesis of MPEG-PCL and Mal-PEG-PCL were completed by ring-opening polymerization of $\varepsilon$-caprolactone at $130^{\circ} \mathrm{C}$ overnight, as previously described. ${ }^{24,25}$ In brief, MPEGhydroxyl and Mal-PEG-hydroxyl were used as a macroinitiator, where $\varepsilon$-caprolactone was a monomer and stannous octoate was the catalyst. The final synthesized polymer was recovered by dissolving in tetrahydrofuran followed by precipitation in ice-cooled diethyl ether. The resultant precipitation was filtered and dried at room temperature in a vacuum. The molecular weights of the synthesized polymers - measured by hydrogen-1 nuclear magnetic resonance (Avance III HD; Bruker Corporation, Billerica, MA, USA) using deuterated chloroform as the solvent and gel permeation chromatography 
(Waters 510 HPLC pump/410 differential refractometer; EMD Millipore, Billerica, MA, USA) with a tetrahydrofuran eluent - were previously shown to be 11,566 and 10,850 for MPEG-PCL and Mal-PEG-PCL, respectively. ${ }^{14}$ The size of the micelles was $70 \pm 37 \mathrm{~nm}$, as determined by dynamic light scattering (DelsaNano S, Beckman Coulter, Brea, CA, USA).

\section{Preparation of GRP78BP and DTPA-conjugated micelles}

The modified GRP78BP (RGDGGWIFPWIQLC) ${ }^{14}$ was first thiolated in a 40:1 molar ratio excess of 2-iminothiolane (Traut's reagent) in $0.15 \mathrm{M}$ sodium borate buffer ( $\mathrm{pH} \mathrm{8)}$ supplemented with $1 \mathrm{mM}$ ethylenediaminetetraacetic acid for 1 hour, as described previously. ${ }^{26}$ The buffer was then exchanged with $0.15 \mathrm{M}$ sodium chloride ( $\mathrm{pH} 7$ ) containing $1 \mathrm{mM}$ ethylenediaminetetraacetic acid using a $1 \mathrm{kDa}$ cutoff dialysis tube (GE Healthcare, Little Chalfont, UK), and the thiolated peptide was concentrated to a volume of $50 \mu \mathrm{L}$. The thiolated peptide was then mixed with Mal-terminated micelles at a thiolated peptide/Mal molar ratio of 1:5. The mixture was stirred at room temperature for 2 hours. The unconjugated peptide was removed through Sephadex ${ }^{\circledR}$ G-25 column (GE Healthcare) and eluted with $0.15 \mathrm{M}$ sodium chloride buffer ( $\mathrm{pH} 7$ ). The concentration of peptide in each collected fraction was analyzed by $\mathrm{BCA}^{\mathrm{TM}}$ protein assay (Thermo Fisher Scientific, Waltham, MA, USA).

On the other hand, fluorenylmethyloxycarbonyl chloride-PEG-PCL was deprotected by stirring in $2 \mathrm{~mL}$ of $20 \%$ piperidine in dimethylformamide for 2 hours at room temperature. The resultant amine $\left(\mathrm{NH}_{2}\right)$-modified PEG-PCL was then purified using dialysis in distilled water for 7 days and conjugated with DTPA dianhydride, as described previously. ${ }^{27}$ Finally, tetrahydrofuran was used as the eluent at a flow rate of $1.0 \mathrm{~mL} / \mathrm{minute}$. The success of DTPA-PEG-PCL production was analyzed by matrix-assisted laser desorption/ionization time-of-flight mass spectrometry (MALDI-TOF; Bruker), and the DTPA conjugation efficiency with ${ }^{111}$ In was evaluated by the radiolabeling yields of ${ }^{111}$ In-DTPA-PEG-PCL as analyzed by instant thin layer chromatography (AR-2000 radio-TLC Imaging Scanner, Bioscan, France).

\section{In vivo nuclear imaging in GC xenografts}

The mice received ${ }^{111}$ In-labeled micelles with or without GRP78BP conjugation via intravenous injection $(n=3$ for

${ }^{111}$ In-labeled micelles, $\mathrm{n}=3$ for GRP78BP ${ }^{111}$ In-labeled micelles). Each mouse was injected with $1 \mathrm{mCi}$ of ${ }^{111} \mathrm{In}$.
The distribution of ${ }^{111}$ In-labeled micelles in the mice bearing MKN45 tumors was evaluated by nanoSPECT/CT images at 24 hours and 48 hours after the micelles were intravenously injected. The radioactive intensity was measured against the baseline, ie, the radioactive intensity of a muscle from a leg without the tumor embedded.

\section{Statistical analysis}

Statistical software Prism ${ }^{\circledR} 5$ (GraphPad Software, Inc, La Jolla, CA, USA) was used to calculate the significance according to Student's $t$-test. Significance was set at $P<0.05$.

\section{Results}

\section{Construction of GRP78-guided II'In-labeled polymeric micelles}

It was previously shown that GRP78 is overexpressed on the surface of GC tissues, ${ }^{14}$ indicating that it can be used as a reliable target for GC diagnosis. In the current study, GRP78BP-guided micelles conjugated with DTPA - which can specifically trap the radioisotope ${ }^{111} \mathrm{In}$ - were manufactured to detect the GCs in a xenograft mouse model. The construct of the micelle-based nuclear imaging agent is illustrated in Figure 1. In order to investigate and compare the probing efficacy of GRP78BP in nuclear imaging, another report agent lacking GRP78BP was also created.

Initially, the lipidic micelles were synthesized, as previously described, ${ }^{15,24}$ and then labeled with GRP78BP, ${ }^{14}$ which was purified by a Sephadex G25 column. Compared to the elution of free GRP78BP from fraction seven to ten, the GRP78BP-conjugated micelles were eluted in fraction four (Figure 2A). This simple column was also used to exclude the unlabeled peptides. To trap ${ }^{111}$ In onto micelles, the synthesized micelles were first modified in the N-terminal by adding $\mathrm{NH}_{2}$ and then labeled with DTPA for chelating the radioisotopes, as previously described. ${ }^{27}$ The three kinds of micelles were detected and analyzed using matrix-assisted laser desorption ionization time-of-flight mass spectrometry to determine the successful rate of conjugations. The results from Figure 2B reveal that the mass/charge ratio of fluorenylmethyloxycarbonyl chloride-PEG-PCL was 5780.97, which was speculated to be the molecule with $2 \mathrm{H}^{+}$ according to a previous measurement using nuclear magnetic resonance. ${ }^{14}$ The mass/charge ratio of $\mathrm{NH}_{2}-\mathrm{PEG}-\mathrm{PCL}$ and DTPA-PEG-PCL was 6185.65 and 6585.45, respectively. The results suggest that DTPA was successfully conjugated with PEG-PCL. Next, Mal-PEG-PCL and DTPA-PEGPCL were mixed together to form the polymeric micelles, which were then labeled with GRP78BP (Figure 1A). 
A

111 In/micelles

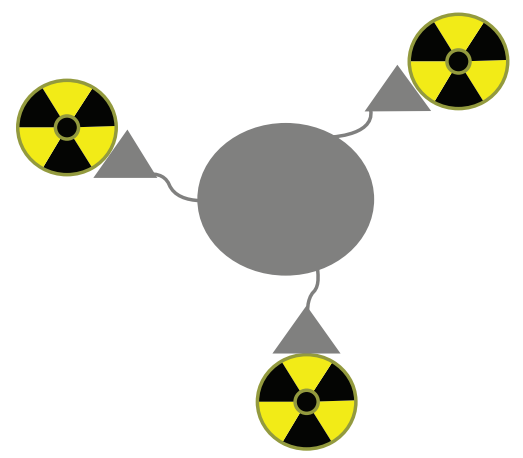

B $\quad$ GRP78BP-111 In/micelles

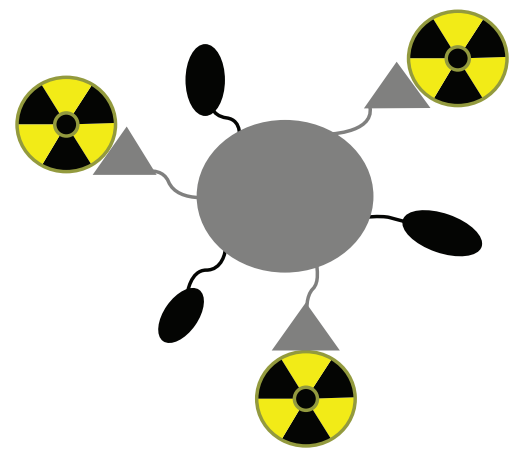

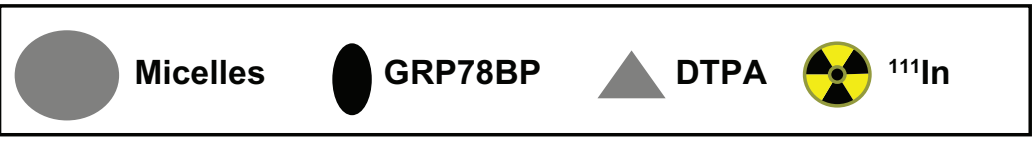

Figure I Schematic design of a nuclear imaging agent for detecting gastric cancer. (A) Polymeric micelles were labeled with radioactive isotope "'In through a DTPA molecule and then (B) conjugated with GRP78BP (RGDKGGWIFPWIQL).

Abbreviations: DTPA, diethylenetriaminepentaacetic acid; GRP78BP, glucose-regulated protein 78 binding peptide; "'In, indium-III.

\section{Tumor detection using nuclear imaging}

To ensure the labeling efficacy of ${ }^{111}$ In onto the micelles, instant thin layer chromatography was performed to separate ${ }^{111} \mathrm{In}$ and ${ }^{111} \mathrm{In}$-labeled micelles in $10 \mathrm{~cm}$ membrane electrophoresis. A $\gamma$-counter was used to detect the radioactive signals. The ${ }^{111}$ In labeling efficacy with or without GRP78BP micelles was $\sim 90 \%$ (Figure 3), demonstrating that the micelle-based nuclear imaging agent was synthesized with stable conjugation in phosphate buffered saline.

In a previous study, it was found that both GRP78BP and micelles displayed no significant in vitro cytotoxic effects in MKN45 cells. ${ }^{14}$ In addition to the measurement of cytotoxicity of the imaging agents, the expression level of GRP78 in GCs and other forms of cancer cells was also measured. The expression level of GRP78 was significantly increased in GC cells (AGS and MKN45), colorectal cancer cells (HCT-15), and pancreatic cancer cells (AR42J), but relatively lower in breast cancer cells (MCF-7), liver cancer cells (HepG2), and prostate cancer cells (PC-3) (Figure 4A), suggesting that GC cells can express a high level of GRP78 on the cell membrane. Therefore, GRP78 is a good candidate for a GC biomarker. Due to the difficulty in generating a xenograft murine model bearing AGS GC tumors, the MKN45 cell line was selected for establishment of the xenograft animal model.

Next, the ${ }^{111}$ In-labeled micelles with or without GRP78BP were administered via tail vein injection into the GC xenografts. To trace the accumulation of micelles, the $\gamma$-signals 24 hours after administration were detected. It was observed that micelles with or without GRP78BP can accumulate in several organs including the liver, spleen, and tumor (Figure 4B). However, GRP78BP-labeled micelles displayed a higher accumulation of $\gamma$-signals $(P<0.05$; Figure 4B and C) in the tumor, indicating that GRP78BP can guide the micelles into tumor tissue. The results indicate that the biomarker probe GRP78BP can improve the intensity of detection signals, leading to increased diagnostic accuracy.

\section{Discussion}

A novel nuclear imaging agent for GC diagnosis was designed and developed using carrier NPs containing combined polymeric micelles, specific tumor-targeted GRP78BP, and a DTPA $-{ }^{111}$ In complex. NPs are widely used in tumor treatment (eg, nuclear imaging, tumor therapy) because of their enhanced permeability and retention effect and subsequent increased accumulation on the surface of tumors. ${ }^{27-30}$ In this study, it was observed that the conjugated GRP78BP can direct micelles to tumor tissues, thus improving the accumulation efficacy in tumors. This novel construct can enlarge the impact of NPs in the diagnosis of solid tumors.

Compared to other tumor-targeting probes (eg, specific antibodies), a simple form of peptide molecule was synthesized with less difficulty and lower cost. ${ }^{31-33}$ However, peptides had shorter in vivo half-life due to rapid clearance after intravenous administration. No difference in accumulation rate was found when using isotope rhenium-188conjugated GRP78BP, and the total amount of radioactive signal dramatically decreased with time (data not shown), indicating that the peptide was rapidly cleared in animals. 
A

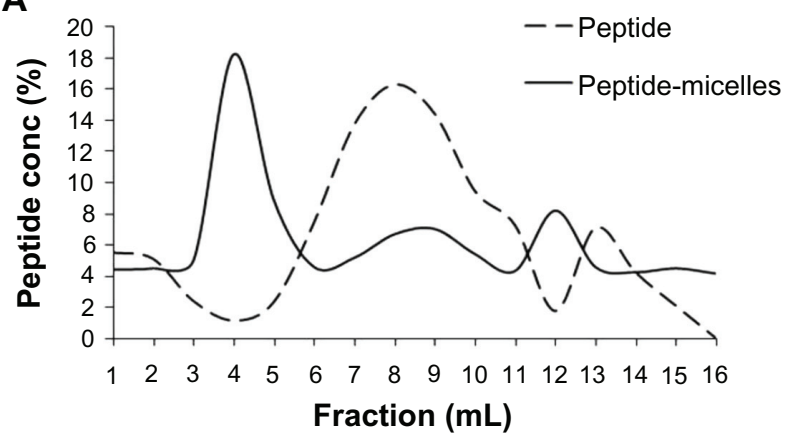

B

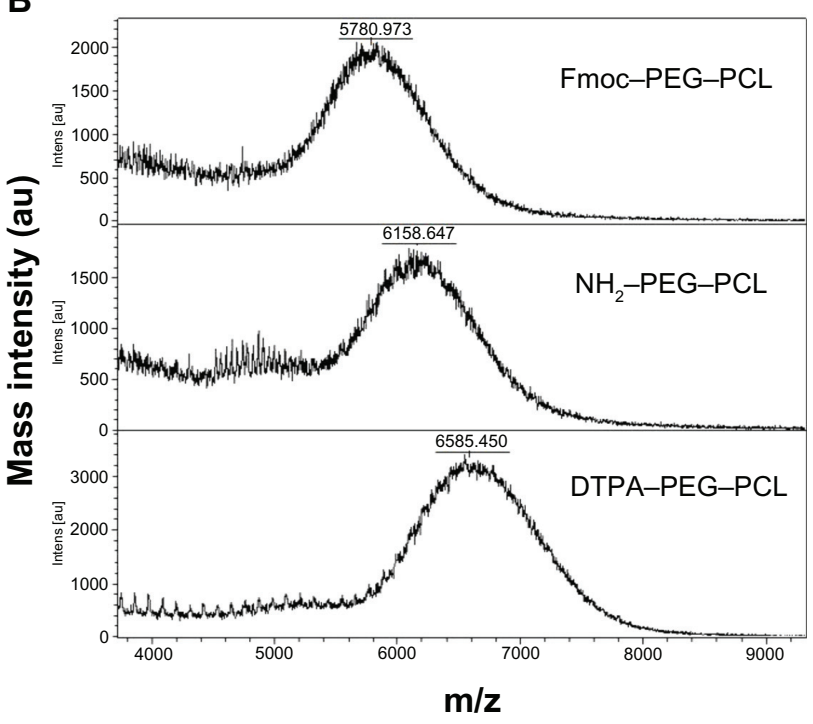

Figure 2 Verification of the conjugation of glucose-regulated protein 78 binding peptide or DTPA with polymeric micelles. (A) Glucose-regulated protein 78 binding peptide conjugated with micelles was purified by Sephadex ${ }^{\circledR}$ G25 column (GE Healthcare, Little Chalfont, UK) in elution fraction four with phosphate buffered saline. (B) Fmoc-PEG-PCL, $\mathrm{NH}_{2}$-modified PEG-PCL, and DTPA-conjugated PEG$\mathrm{PCL}$ were analyzed using matrix-assisted laser desorption/ionization time-of-flight mass spectrometry.

Note: The molecular weights of the above three compounds were increased from a mass/charge ratio of 5780.973 to 6585.450 , indicating successful conjugation.

Abbreviations: DTPA, diethylenetriaminepentaacetic acid; Fmoc, fluorenylmethyloxycarbonyl chloride; $\mathrm{NH}_{2}$, amine; $\mathrm{PCL}$, poly( $(\varepsilon$-caprolactone); $\mathrm{PEG}$, poly(ethylene glycol); conc, concentration; intens, intensity.

Therefore, it was speculated that GRP78BP alone was probably insufficient to use as an application for nuclear imaging because of the rapid metabolic rate of peptides. Nevertheless, GRP78BP can recognize and bind to the overexpressed surface biomarker of GC, GRP78, ${ }^{20}$ and therefore it was used as a guiding probe to direct the NPs targeting to tumor tissue. The in vivo nuclear imaging was recorded, which indicated that GRP78 ${ }^{111}$ In-labeled micelles are a potential tool for the diagnosis of GC.

To characterize in vivo imaging and assess the targeting efficacy, the tumor xenografts were embedded into the legs of nude mice, and then images were measured and analyzed using a nanoSPECT/CT instrument. The results demonstrate
A

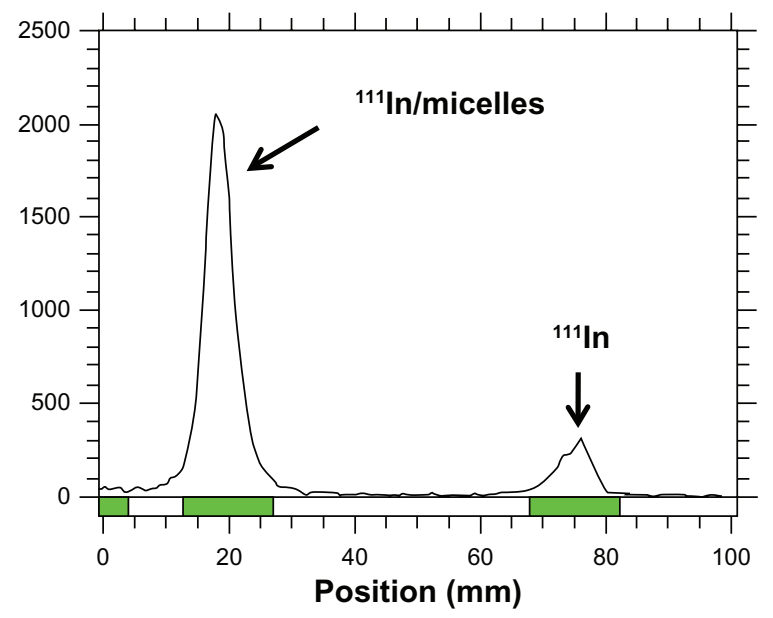

B

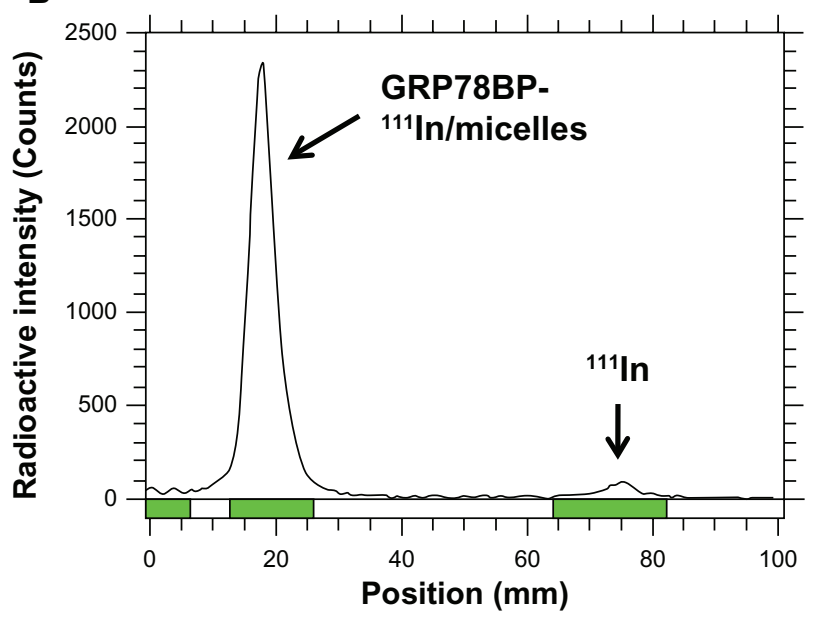

Figure 3 '"'In labeled with diethylenetriaminepentaacetic acid-conjugated micelles. The diethylenetriaminepentaacetic acid-conjugated micelles with or without GRP78BP conjugation were labeled with "'In in phosphate buffered saline for I hour. Instant thin layer chromatography was performed to measure the radio-labeling efficiency, which was $85 \%$ and $93 \%$ for (A) '"'In-labeled micelles and (B) GRP78BP "'In-labeled micelles, respectively.

Note: The products without purification were further performed in nuclear imaging experimentation.

Abbreviations: GRP78BP, glucose-regulated protein 78 binding peptide; '"'In, indium-III.

that micelles with or without GRP78BP were largely retained in the liver and spleen, although GRP78BP improved the retention efficacy in tumors. In the clinical diagnosis of GC, the obtained radioactive signal derived from the liver may interfere with positive nuclear imaging for GC and impair the diagnostic accuracy. However, with the combined tool of SPECT/CT, the spatial location of the stomach can be distinguished from the liver using three-dimensional rotation with high resolution to remove the false signaling. Previous studies also indicated that SPECT can be used as a noninvasive tool for detecting gastric accommodation. ${ }^{34,35}$ The advantage of using nanoSPECT/CT to monitor images is being able to distinguish the planar overlapped signals 

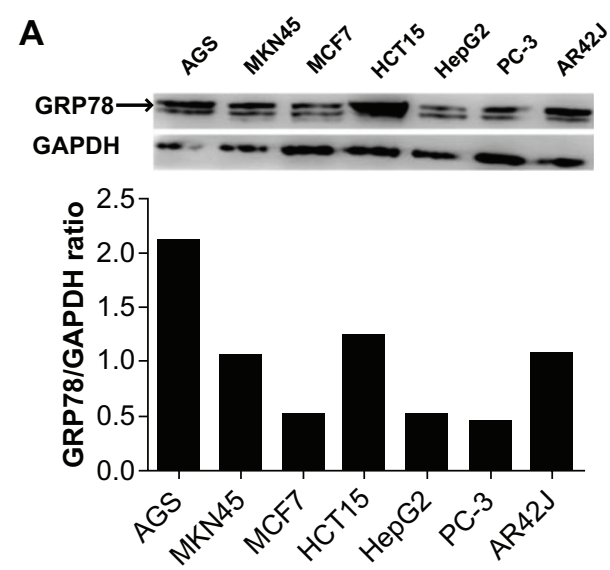

B

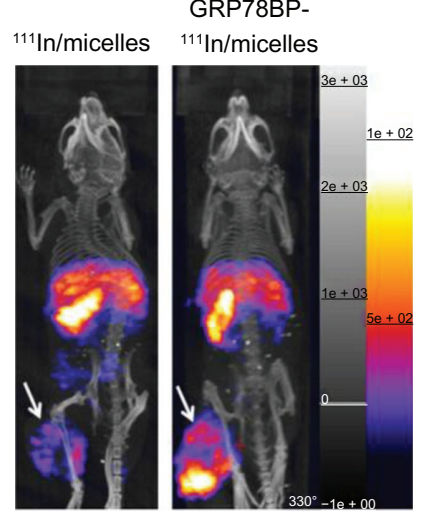

C

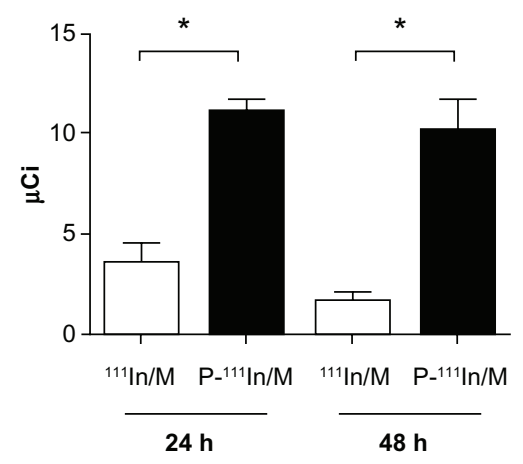

Figure 4 GRP78 specifically expressed in gastric cancer cells and GRP78BP improved the tumor-accumulated efficacy of micelle polymers. (A) GRP78 expression in the variety of cancer cell lines was detected using Western blotting. The results demonstrate a higher expression of GRP78 in gastric cancer cells (AGS and MKN45), colorectal cancer cells (HCT-I5), and pancreatic cancer cells (AR42J), but lower expression in breast cancer cells (MCF-7), liver cancer cells (HepG2), and prostate cancer cells (PC-

3). Therefore, it was found that GRP78 is specifically expressed in gastric cancer. (B) Radioactive compounds ("'In-labeled micelles, GRP78BP I'IIn-labeled micelles, and I'In alone) were injected into gastric cancer xenografts in mice, while '"'In was used as reference for radioactive quantification. From the nuclear imaging captured by nano single photon emission computed tomography/computed tomography, it was found that the radioactive intensity of GRP78BP I'IIn-labeled micelles was higher than that of I'IIn-labeled micelles in the tumor (indicated by arrows), demonstrating that GRP78BP can guide the micelles into tumor cells and increase the radioactive signal. (C) Radio quantification at 24 hours and 48 hours showed that GRP78BP (P) improved the accumulated efficacy of micelles in tumor tissue compared to ${ }^{\prime \prime \prime}$ In-labeled micelles. Note: $* P<0.05$.

Abbreviations: GAPDH, glyceraldehyde 3-phosphate dehydrogenase; GRP78, glucose-regulated protein 78; GRP78BP, glucose-regulated protein 78 binding peptide; I'In, indium-III; M, micelles.

derived from three-dimensional rotation, such as the liver and spleen, which both demonstrated a high radioactive signal derived from partially overlapping organs (Figure 4B). Therefore, polymeric micelles as a form of NPs may be an ideal material in nuclear imaging for GC diagnosis.

NPs also can be linked to a specific targeted GRP78BP or other specific antibodies to improve the tumor-targeted efficacy. The size of synthesized micelles is around $\sim 70 \mathrm{~nm}$, within the range of so-called NPs. The main limitation in the application of polymeric micelles is their high specific accumulation in the liver and spleen. The accumulation of micelles in the liver and spleen may be a result of normal metabolism. This inevitable metabolic character of micelles may lead to a misdiagnosis of metastatic liver tumors migrated from $\mathrm{GC}$ when analysis is made by three-dimensional rotation SPECT/CT. Therefore, other diagnostic tools such as ultrasound should also be considered.

\section{Conclusion}

In this study, ${ }^{111}$ In-labeled polymeric micelles decorated with GRP78BP were rationally designed and constructed to generate a novel nuclear imaging agent during tumor diagnosis. It was demonstrated that GRP78BP-guided ${ }^{111}$ In-labeled micelles can enhance the targeting efficiency, concentrate imaging reagent onto tumors, and provide a better resolution and diagnostic accuracy in nuclear imaging, suggesting it is a novel imaging agent for cancer diagnosis. This study is the first report using nuclear imaging agents to diagnose GCs in vivo. These conjugated micelles, within the size range of NPs, provide a new methodology to monitor and trace GCs with high quality nuclear imaging. It may be useful for $\mathrm{GC}$ diagnosis in the future.

\section{Acknowledgments}

This project was supported by the grant ARA010201 from Atomic Energy Council of Republic of China and by the grant 99TMU-TMUH-03-2 from Taipei Medical University Hospital.

\section{Disclosure}

The authors report no conflicts of interest in this work.

\section{References}

1. Amin A, Gilmour H, Graham L, Paterson-Brown S, Terrace J, Crofts TJ. Gastric adenocarcinoma missed at endoscopy. J R Coll Surg Edinb. 2002;47(5):681-684.

2. Liu G, Swierczewska M, Lee S, Chen X. Functional nanoparticles for molecular imaging guided gene delivery. Nano Today. 2010;5(6): 524-539.

3. Jambor I, Borra R, Kemppainen J, et al. Functional imaging of localized prostate cancer aggressiveness using 11C-acetate PET/CT and 1H-MR spectroscopy. J Nucl Med. 2010;51(11):1676-1683.

4. Seitz M, Shukla-Dave A, Bjartell A, et al. Functional magnetic resonance imaging in prostate cancer. Eur Urol. 2009;55(4):801-814.

5. Kim JK, Jang YJ, Cho G. Multidisciplinary functional MR imaging for prostate cancer. Korean J Radiol. 2009;10(6):535-551.

6. Zhang R, Xiong C, Huang M, et al. Peptide-conjugated polymeric micellar nanoparticles for dual SPECT and optical imaging of EphB4 receptors in prostate cancer xenografts. Biomaterials. 2011;32(25):5872-5879. 
7. Kjaer A. Molecular imaging of cancer using PET and SPECT. Adv Exp Med Biol. 2006;587:277-284.

8. Seo Y, Franc BL, Hawkins RA, Wong KH, Hasegawa BH. Progress in SPECT/CT imaging of prostate cancer. Technol Cancer Res Treat. 2006;5(4):329-336.

9. Feng X, Lv F, Liu L, et al. Conjugated polymer nanoparticles for drug delivery and imaging. ACS Appl Mater Interfaces. 2010;2(8): 2429-2435.

10. Katanasaka Y, Ishii T, Asai T, et al. Cancer antineovascular therapy with liposome drug delivery systems targeted to BiP/GRP78. Int J Cancer. 2010;127(11):2685-2698.

11. Monsky WL, Fukumura D, Gohongi T, et al. Augmentation of transvascular transport of macromolecules and nanoparticles in tumors using vascular endothelial growth factor. Cancer Res. 1999;59(16): 4129-4135.

12. Peng $\mathrm{C}$, Zheng L, Chen Q, et al. PEGylated dendrimer-entrapped gold nanoparticles for in vivo blood pool and tumor imaging by computed tomography. Biomaterials. 2012;33(4):1107-1119.

13. Jin Y, Ren X, Wang W, et al. A 5-fluorouracil-loaded $\mathrm{pH}$-responsive dendrimer nanocarrier for tumor targeting. Int J Pharm. 2011;420(2): 378-384.

14. Cheng CC, Lu N, Peng CL, et al. Targeting to overexpressed glucoseregulated protein 78 in gastric cancer discovered by 2D DIGE improves the diagnostic and therapeutic efficacy of micelles-mediated system. Proteomics. 2012;12(15-16):2584-2597.

15. Peng CL, Shih YH, Lee PC, Hsieh TM, Luo TY, Shieh MJ. Multimodal image-guided photothermal therapy mediated by $188 \mathrm{Re}$-labeled micelles containing a cyanine-type photosensitizer. ACS Nano. 2011; 5(7):5594-5607.

16. Hong GB, Zhou JX, Yuan RX. Folate-targeted polymeric micelles loaded with ultrasmall superparamagnetic iron oxide: combined small size and high MRI sensitivity. Int J Nanomedicine. 2012;7:2863-2872.

17. Cai LL, Liu P, Li X, et al. RGD peptide-mediated chitosan-based polymeric micelles targeting delivery for integrin-overexpressing tumor cells. Int J Nanomedicine. 2011;6:3499-3508.

18. Oerlemans C, Bult W, Bos M, Storm G, Nijsen JF, Hennink WE. Polymeric micelles in anticancer therapy: targeting, imaging and triggered release. Pharm Res. 2010;27(12):2569-2589.

19. Blanco E, Kessinger CW, Sumer BD, Gao J. Multifunctional micellar nanomedicine for cancer therapy. Exp Biol Med (Maywood). 2009; 234(2):123-131.

20. Arap MA, Lahdenranta J, Mintz PJ, et al. Cell surface expression of the stress response chaperone GRP78 enables tumor targeting by circulating ligands. Cancer Cell. 2004;6(3):275-284.

21. Lee AS. GRP78 induction in cancer: therapeutic and prognostic implications. Cancer Res. 2007;67(8):3496-3499.
22. Shieh MJ, Hsu CY, Huang LY, Chen HY, Huang FH, Lai PS. Reversal of doxorubicin-resistance by multifunctional nanoparticles in MCF-7/ ADR cells. J Control Release. 2011;152(3):418-425.

23. Krishna AD, Mandraju RK, Kishore G, Kondapi AK. An efficient targeted drug delivery through apotransferrin loaded nanoparticles. PLoS One. 2009;4(10):e7240.

24. Peng CL, Shieh MJ, Tsai MH, Chang CC, Lai PS. Self-assembled star-shaped chlorin-core poly(E-caprolactone)-poly(ethylene glycol) diblock copolymer micelles for dual chemo-photodynamic therapies. Biomaterials. 2008;29(26):3599-3608.

25. Peng CL, Lai PS, Lin FH, Yueh-Hsiu Wu S, Shieh MJ. Dual chemotherapy and photodynamic therapy in an HT-29 human colon cancer xenograft model using SN-38-loaded chlorin-core star block copolymer micelles. Biomaterials. 2009;30(21):3614-3625.

26. Olivier JC, Huertas R, Lee HJ, Calon F, Pardridge WM. Synthesis of pegylated immunonanoparticles. Pharm Res. 2002;19(8):1137-1143.

27. Fonge H, Lee H, Reilly RM, Allen C. Multifunctional block copolymer micelles for the delivery of 111In to EGFR-positive breast cancer cells for targeted Auger electron radiotherapy. Mol Pharm. 2010;7(1): $177-186$.

28. Shiraishi K, Kawano K, Minowa T, Maitani Y, Yokoyama M. Preparation and in vivo imaging of PEG-poly(L-lysine)-based polymeric micelle MRI contrast agents. J Control Release. 2009;136(1):14-20.

29. Lammers T, Rizzo LY, Storm G, Kiessling F. Personalized nanomedicine. Clin Cancer Res. 2012;18(18):4889-4894.

30. Namiki Y, Fuchigami T, Tada N, et al. Nanomedicine for cancer: lipidbased nanostructures for drug delivery and monitoring. Acc Chem Res. 2011;44(10):1080-1093.

31. Behera A, Banerjee I, De K, et al. Synthesis, characterization, conformational analysis of a cyclic conjugated octreotate peptide and biological evaluation of (99m)Tc-HYNIC-His (3)-octreotate as novel tracer for the imaging of somatostatin receptor-positive tumors. Amino Acids. 2013;44(3):933-946.

32. Abiraj K, Mansi R, Tamma ML, et al. Bombesin antagonist-based radioligands for translational nuclear imaging of gastrin-releasing peptide receptor-positive tumors. J Nucl Med. 2011;52(12):1970-1978.

33. Okarvi SM. Peptide-based radiopharmaceuticals and cytotoxic conjugates: potential tools against cancer. Cancer Treat Rev. 2008; 34(1):13-26.

34. Bouras EP, Delgado-Aros S, Camilleri M, et al. SPECT imaging of the stomach: comparison with barostat, and effects of sex, age, body mass index, and fundoplication. Single photon emission computed tomography. Gut. 2002;51(6):781-786.

35. Kim DY, Camilleri M. Noninvasive measurement of gastric accommodation by SPECT. Korean J Intern Med. 2002;17(1):1-6.
International Journal of Nanomedicine

\section{Publish your work in this journal}

The International Journal of Nanomedicine is an international, peerreviewed journal focusing on the application of nanotechnology in diagnostics, therapeutics, and drug delivery systems throughout the biomedical field. This journal is indexed on PubMed Central, MedLine, CAS, SciSearch ${ }^{\circledR}$, Current Contents ${ }^{\circledR} /$ Clinical Medicine,

\section{Dovepress}

Journal Citation Reports/Science Edition, EMBase, Scopus and the Elsevier Bibliographic databases. The manuscript management system is completely online and includes a very quick and fair peer-review system, which is all easy to use. Visit http://www.dovepress.com/ testimonials.php to read real quotes from published authors. 